\title{
Correlation analysis of mRNA expression and prognosis of hOGG1 gene polymorphism in patients with non-small cell lung cancer
}

\author{
JING WANG $^{1 *}$ and PUZHAO WU ${ }^{2 *}$ \\ Departments of ${ }^{1}$ Oncology and ${ }^{2}$ Interventional Oncology, Xiang Yang No. 1 People's Hospital, \\ Hubei University of Medicine, Xiangyang, Hubei 441000, P.R. China
}

Received September 18, 2018; Accepted May 22, 2019

DOI: 10.3892/ol.2019.10586

\begin{abstract}
Level of mRNA expression and gene polymorphism of human 8-hydroxyguanine glycosidase 1 (hOGG1) in patients with non-small cell lung cancer (NSCLC) were investigated. A polymorphism analysis of hOGG1 gene rs1052133 locus in 182 NSCLC patients (NSCLC group) surgically treated in Xiang Yang No. 1 People's Hospital, Hubei University of Medicine from January 2008 to January 2012 and 200 healthy individuals (control group) was performed. The expression level of hOGG1 was compared between cancer tissues and adjacent tissues of NSCLC patients, and the survival rate was analyzed. The expression level of hOGG1 was significantly higher in cancer tissues than that in adjacent tissues $(\mathrm{P}<0.001)$. Taqman probe method was used to detect the genotypes of hOGG1 polymorphism locus rs1052133, with the genotype distribution frequencies of NSCLC group $(\mathrm{P}=0.411)$ and control group $(\mathrm{P}=0.354)$ consistent with the Hardy-Weinberg equilibrium. The proportion of $\mathrm{C} / \mathrm{C}$ gene was significantly higher in NSCLC group than that in control group $(\mathrm{P}=0.008, \mathrm{OR}=2.2,95 \%, \mathrm{CI}=1.27-4.52)$. The median value of the hOGG1 expression level in detection results as the boundary, NSCLC patients were divided into hOGG1 high expression group $(\geq 3.61)$ with 91 cases and hOGG1 low expression group (<3.61) with 91 cases. The 1-, 2- and 3-year survival rates of patients in hOGG1 low expression group were significantly higher than those in hOGG1 high expression group $(\mathrm{P}=0.007)$. The 3 -year survival rate in hOGG1 low expression group is significantly higher than that
\end{abstract}

Correspondence to: Dr Puzhao Wu, Department of Interventional Oncology, Xiang Yang No. 1 People's Hospital, Hubei University of Medicine, 15 Jiefang Road, Fancheng, Xiangyang, Hubei 441000, P.R. China

E-mail: znaqam@163.com

*Contributed equally

Key words: hOGG1, expression level, gene polymorphism, NSCLC, prognosis in hOGG1 high expression group $(\mathrm{P}=0.007)$. The sensitivity, specificity and AUC of hOGG1 to patient survival prediction were $83.33 \%, 64.29 \%$, and 0.816 , respectively. In conclusion, hOGG1 is highly expressed in NSCLC tissues. Compared to $\mathrm{S} / \mathrm{S}$ and $\mathrm{S} / \mathrm{C}$ genotypes, the $\mathrm{C} / \mathrm{C}$ gene was found to be more common in NSCLC group than in control group. Thus, hOGG1 has a high predictive value for patient survival.

\section{Introduction}

Lung cancer is one of main malignant tumors that cause death in cancer patients, accounting for $10-13 \%$ of systemic malignant tumors, with non-small cell lung cancer (NSCLC) accounting for $80-85 \%$ of all lung cancers $(1,2)$. Early NSCLC has no obvious specific clinical manifestations, so it is often ignored by patients who then miss the best treatment period, leading to high prognostic mortality of NSCLC (3). According to Gettinger et al (4), approximately $78 \%$ of NSCLC patients are in the middle or advanced stage when diagnosed, losing the chance of surgical cure. Planchard et al (5) reported that NSCLC patients have a 5-year survival rate of only $41.8 \%$. At present, chemotherapy is the main non-surgical medical treatment for NSCLC. The clinical treatment of NSCLC chemotherapy is mainly to prolong the survival time of patients and improve their quality of life (6). Due to its high incidence and mortality, NSCLC is a hot research topic in the clinic.

The human 8-hydroxyguanine glycosidase 1 (hOGG1) gene is a DNA oxidative damage repair gene (7). It can recognize and specifically excise the oxidative damage product of DNA, 8-hydroxydeoxyguanine, repairing damaged DNA (8). The hOGG1 gene has a C/G polymorphism in the 1245 th base of exon 7 , making the 326 th codon produce serine (S) or cysteine (C), resulting in $\mathrm{S} \rightarrow \mathrm{C}$ amino acid replacement $(9)$. Related literature has reported $(10,11)$ that the mutation of base $\mathrm{C}$ to $\mathrm{G}$ will lead to a decrease in the activity of hOGG1 enzyme, making the body susceptible to diseases such as tumors. Studies have reported (12) that the hOGG1 Ser326Cys polymorphism increases the susceptibility of high-risk prostates. However, currently, there are less related literature on the hOGG1 Ser326Cys (rs1052133) gene polymorphism and the prognosis in NSCLC patients. The aim of this study was to investigate the mRNA expression level 
Table I. Patient basic data of NSCLC group and control group [n (\%)].

\begin{tabular}{|c|c|c|c|c|}
\hline Factors & NSCLC group $(n=182)$ & Control group $(n=200)$ & $\chi^{2}$ value & $\mathrm{P}$-value \\
\hline Sex & & & 0.105 & 0.747 \\
\hline Male & $104(57.14)$ & $111(55.50)$ & & \\
\hline Female & $78(42.86)$ & $89(44.50)$ & & \\
\hline Age (years) & & & 2.318 & 0.128 \\
\hline$<40$ & $85(46.70)$ & $119(59.50)$ & & \\
\hline$\geq 40$ & $97(53.30)$ & $81(40.50)$ & & \\
\hline Smoking & & & 0.384 & 0.536 \\
\hline Yes & $98(53.85)$ & $114(57.00)$ & & \\
\hline No & $84(46.15)$ & $86(43.00)$ & & \\
\hline Drinking & & & 0.025 & 0.876 \\
\hline Yes & $75(41.21)$ & $84(42.00)$ & & \\
\hline No & $107(58.79)$ & $116(58.00)$ & & \\
\hline Chronic diseases & & & 0.153 & 0.696 \\
\hline Yes & $81(44.51)$ & $93(46.50)$ & & \\
\hline No & $101(55.49)$ & $107(53.50)$ & & \\
\hline Pathological diagnosis typing & & & - & - \\
\hline Squamous cell carcinoma & $93(51.10)$ & - & & \\
\hline Adenocarcinoma & $54(29.67)$ & - & & \\
\hline Large cell carcinoma & $35(19.23)$ & - & & \\
\hline
\end{tabular}

NSCLC, non-small cell lung cancer.

and gene polymorphism of hOGG1 in NSCLC patients and analyze the prognostic significance.

\section{Patients and methods}

Patient data. A total of 182 NSCLC patients surgically treated in Xiang Yang No. 1 People's Hospital, Hubei University of Medicine (Xiangyang, China) from January 2008 to January 2012 were the NSCLC group, including 104 males and 78 females, aged 26-71 years, with an average age of $52.57 \pm 2.75$ years. Two hundred healthy individuals in Xiang Yang No. 1 People's Hospital, Hubei University of Medicine were the control group, including 111 males and 89 females, aged 25-67 years, with an average age of 54.16 \pm 3.04 years. All the patients had complete clinical and follow-up data. Electrocardiogram, blood routine and liver and kidney function of patients were normal. Patients with communication impairment or cognitive dysfunction were excluded. All the patients and their families signed an informed consent form and cooperated with medical staff to complete relevant medical treatment. It was found that there was no significant difference in terms of sex, age, smoking, drinking and chronic diseases between the two groups $(\mathrm{P}>0.05)$, indicating that the experimental results are accurate and credible. Details of subject basic data are shown in Table I. This study was approved by Xiang Yang No. 1 People's Hospital, Hubei University of Medicine.

Extraction of total DNA and RNA. Fasting venous blood (5 ml) was collected from patients in NSCLC group and control group into EDTA anticoagulant tubes. The DNA in the blood was extracted strictly according to the instructions of the animal tissue/cell genomic DNA extraction kit (Shanghai Jingke Chemical Technology Co., Ltd., item no.: D1700). Cancer tissues and adjacent tissues surgically resected from patients in NSCLC group were added to the total RNA extraction reagent (Shanghai Kanglang Biotechnology Co., Ltd., item no.: KL101-151) to extract the RNA in tissues. The UV-9000S ultraviolet spectrophotometer (Jingfeile Instrument Co., Ltd.) was used to measure the quality and concentration of DNA and RNA at wavelengths of 260 and $280 \mathrm{~nm}$. At $\mathrm{OD}_{260} / \mathrm{OD}_{280}$ value of approximately 1.8 the extracted DNA and RNA purity was considered satisfactory, when necessary, re-extraction and identification were performed.

$R T-q P C R$ detection of hOGGl expression. RT-PCR was used to detect the hOGG1 expression, and TRIzol kit (Wuhan Purity Biotechnology Co., Ltd., item no.: CD-13433-ML) to extract whole blood total RNA in strict accordance with the instructions. After the detection of nucleic acid concentration, the instructions of miRNA reverse transcription kit (Beijing Baiaolaibo Technology Co., Ltd., item no.: ALH266-PTO) was used for reverse transcription to generate cDNA. qPCR was performed, with $\beta$-actin as an internal reference, according to the PCR kit (Beijing Zhijiefangyuan Technology Co., Ltd., item no.: RR047A) instructions. qPCR reaction conditions: Pre-denaturation at $95^{\circ} \mathrm{C}$ for $3 \mathrm{~min}$, and then $95^{\circ} \mathrm{C}$ for $30 \mathrm{sec}$, $58^{\circ} \mathrm{C}$ for $30 \mathrm{sec}, 72^{\circ} \mathrm{C}$ for $30 \mathrm{sec}$, for a total of 35 cycles, and extension at $72^{\circ} \mathrm{C}$ for $10 \mathrm{~min}$. After the reaction was completed, the temperature was raised to $95^{\circ} \mathrm{C}$, then reduced to $60^{\circ} \mathrm{C}$; then the temperature was gradually heated to $95^{\circ} \mathrm{C}$ in $20 \mathrm{~min}$, then 
Table II. hOGG1 primer and internal reference sequences.

\begin{tabular}{llll}
\hline Primer sequence & Upstream primer & Downstream primer & Length (bp) \\
\hline hOGG1 & 5'-GGAAGGTGCTTGGGGAAT-3' & 5'-ACTGTCACTAGTCTCACCAG-3' & 200 \\
$\beta$-actin & 5'-ATCATGTTTGAGACCTTCAACA-3' & 5'-CATCTCTTGCTCGAAGTCCA-3' & 348 \\
\hline
\end{tabular}

hOGG1, human 8-hydroxyguanine glycosidase 1.

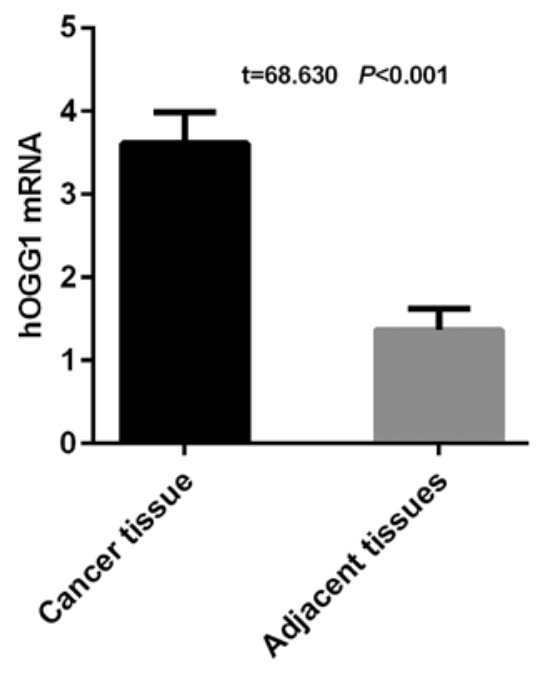

Figure 1. Comparison of hOGG1 expression between cancer tissues and adjacent tissues of NSCLC patients. The results of RT-qPCR detection showed that the expression level of hOGG1 was $3.61 \pm 0.38$ in cancer tissues and $1.37 \pm 0.25$ in adjacent tissues in NSCLC group of patients. That of hOGG1 was significantly higher in cancer tissues than that in adjacent tissues in NSCLC group of patients, with a statistically significant difference $(t=68.630, P<0.001)$. hOGG1, human 8-hydroxyguanine glycosidase 1; NSCLC, non-small cell lung cancer.

maintained for $15 \mathrm{sec}$. ABI 7900 PCR instrument was used for qPCR detection. The primer sequence of hOGG1 was designed and synthesized by Shanghai Jima Pharmaceutical Technology Co., Ltd., with $\beta$-actin as a reaction internal reference. Each group of samples was repeated 3 times. $2^{-\triangle C q}$ method was used to analyze the expression level of hOGG1 in the specimen (13). Primer sequences are shown in Table II.

Genotyping. Taqman probe method was used for hOGG1 rs1052133 locus genotyping. The probe was designed and synthesizedbyShanghaiJimaPharmaceuticalTechnologyCo.,Ltd.,with probe sequences of 5'-FAM-CCAATGCCGCCATG-MGB-3' and 5'-HEX-CGCCAATCCCGCCA-MGB-3'. A total of $5 \mu \mathrm{l}$ Mix buffer, $0.5 \mu 1$ upstream primer, $0.5 \mu 1$ downstream primer, probe sequence $(0.25 \mu \mathrm{l}$ each) and 10 ng DNA (Invitrogen; Thermo Fisher Scientific, Inc.; cat. no. 15279011) were added, and deionized water was added to make a total volume of $10 \mu \mathrm{l}$. The American Bole PCR amplification instrument (Zhejiang Tuopuyunnong Technology Co., Ltd.) was used for amplification. PCR reaction conditions: pre-denaturation at $94^{\circ} \mathrm{C}$ for $3 \mathrm{~min}$, at $94^{\circ} \mathrm{C}$ for $30 \mathrm{sec}$; annealing at $61^{\circ} \mathrm{C}$ for $30 \mathrm{sec}$, at $72^{\circ} \mathrm{C}$ for $30 \mathrm{sec}$, for a total of 30 cycles, and then extension at $72^{\circ} \mathrm{C}$ for $10 \mathrm{~min}$. Taq DNA polymerase was purchased from GeneCopoeia.
Statistical analysis. SPSS 19.5 (Beijing Sichuangweida Information Technology Co., Ltd.) software statistical package was used for processing and analyzing data. Measurement data are expressed as mean \pm standard deviation, and analyzed by t-test. Enumeration data were analyzed by Chi-square test. Survival data were analyzed using Kaplan-Meier survival curve and log rank test. ROC curve was used for the value of hOGG1 expression in the survival of patients with NSCLC. The genotype distribution was analyzed by the goodness-of-fit Chi-square test to determine whether it was consistent with the Hardy-Weinberg equilibrium. $\mathrm{P}<0.05$ was considered to indicate a statistically significant difference.

\section{Results}

Comparison of subject general clinical data between NSCLC group and control group. To achieve accurate and credible experimental results, individuals were compared between two groups in terms of sex, age, smoking, drinking and chronic diseases, with no significant difference $(\mathrm{P}>0.05)$, which proves that the two groups of individuals are comparable (Table I).

Comparison of hOGG1 expression between cancer tissues and adjacent tissues of NSCLC patients. The results of RT-qPCR detection of hOGG1 showed that the expression level of hOGG1 was $3.61 \pm 0.38$ in cancer tissues and $1.37 \pm 0.25$ in adjacent tissues in NSCLC group of patients. Expression of hOGG1 was significantly higher in cancer tissues than that in adjacent tissues in NSCLC group of patients, with a statistically significant difference $(\mathrm{t}=68.630, \mathrm{P}<0.001)$ (Fig. 1).

Hardy-Weinberg equilibrium test of hOGGl genotype distribution frequency and distribution of genotypes in each group. The genotypes of hOGG1 polymorphism locus rs1052133 in NSCLC group and control group included S/S, S/C and $\mathrm{C} / \mathrm{C}$ genotypes, with the genotype distribution frequencies of NSCLC group $\left(\chi^{2}=0.732, P=0.411\right)$ and control group $\left(\chi^{2}=0.642, \mathrm{P}=0.354\right)$ consistent with the Hardy-Weinberg equilibrium. In NSCLC group of patients, there were 6 cases of S/S type, 65 cases of S/C type and 111 cases of C/C type. In control group of patients, there were 15 cases of $\mathrm{S} / \mathrm{S}$ type, 90 cases of S/C type and 95 cases of C/C type. Compared to $\mathrm{S} / \mathrm{S}$ and $\mathrm{S} / \mathrm{C}$ genotypes, the $\mathrm{C} / \mathrm{C}$ gene was found to be more common in NSCLC group than in control group $(\mathrm{P}<0.05$, $\mathrm{OR}=2.2,95 \%, \mathrm{CI}=1.27-4.52$ ). Compared with allele $\mathrm{S}$, it was found that allele $\mathrm{C}$ was more common in NSCLC group than in control group $(\mathrm{P}<0.05)$ (Table III). 
Table III. Distribution of polymorphism locus gene and genotypes in each group.

\begin{tabular}{lcrr}
\hline Polymorphism locus & NSCLC group $(\mathrm{n}=182)$ & Control group $(\mathrm{n}=200)$ & $\chi^{2}$ value \\
\hline rs1052133 & & & \\
Genotype & $6(3.30)$ & $15(7.50)$ & 3.241 \\
S/S & $65(35.71)$ & $90(45.00)$ & 5.638 \\
S/C & $111(60.99)$ & $95(47.50)$ & 23.631 \\
C/C & & & $0.072^{\mathrm{a}}$ \\
Allele & $77(21.15)$ & $120(30.00)$ & $0.018^{\mathrm{b}}$ \\
S & $287(78.85)$ & $280(70.00)$ & $0.001^{\mathrm{c}}$ \\
C & & & \\
\hline
\end{tabular}

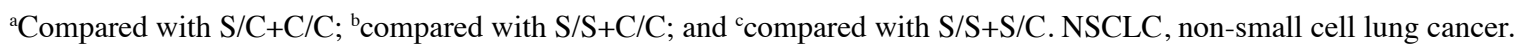

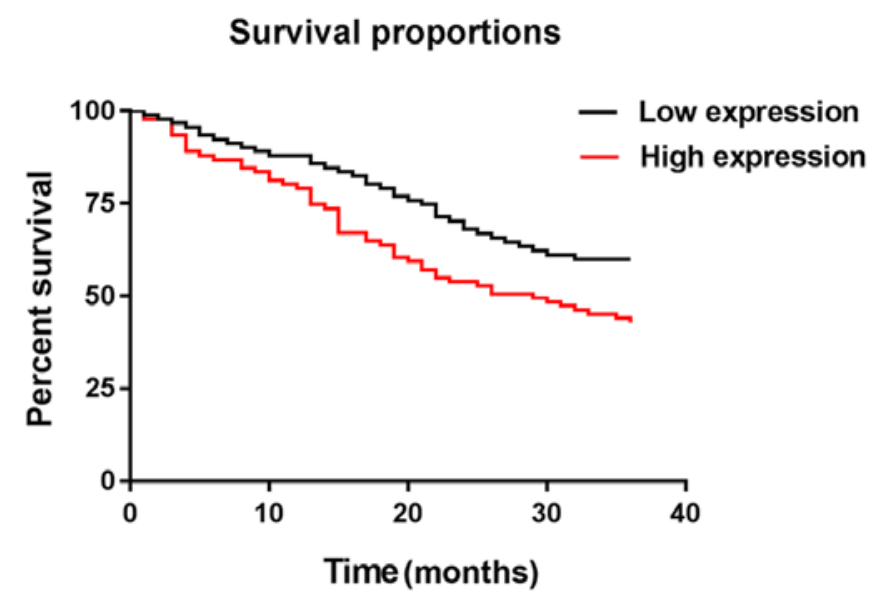

Figure 2. hOGG1 expression and prognosis of NSCLC patients The 1-, 2- and 3 -year survival rates of patients in hOGG1 low expression group were 87.91, 70.33 and $63.74 \%$, respectively. Those of patients in hOGG1 high expression group were $79.12,56.04$, and $43.96 \%$, respectively. Those of patients in hOGG1 low expression group were significantly higher than those in hOGG1 high expression group $(\mathrm{P}=0.007)$. hOGG1, human 8-hydroxyguanine glycosidase 1; NSCLC, non-small cell lung cancer.

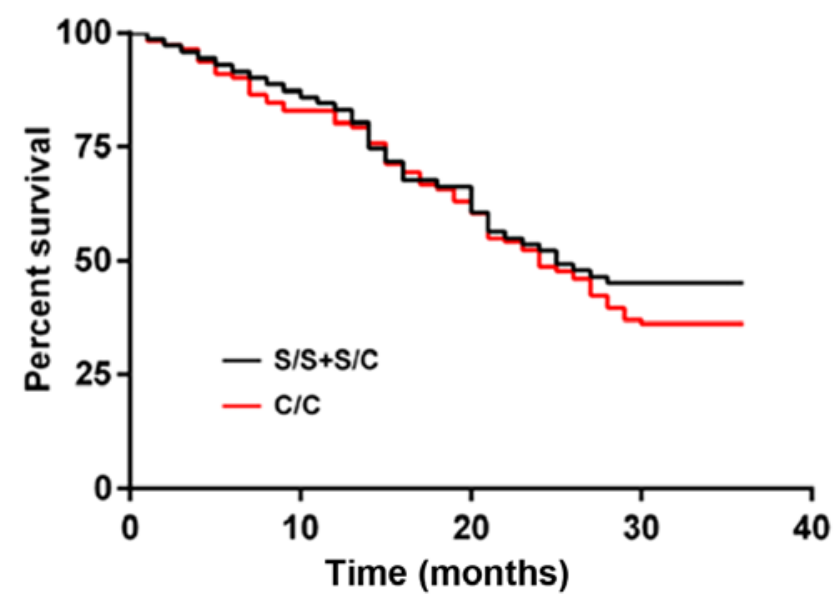

Figure 3. hOGG1 gene polymorphism and prognosis of NSCLC patients. The 1-, 2- and 3-year survival rates of patients with hOGG1 rs1052133 of $\mathrm{S} / \mathrm{S}+\mathrm{S} / \mathrm{C}$ genotypes were $83.10,52.11$, and $45.07 \%$, respectively, and those of patients with $\mathrm{C} / \mathrm{C}$ genotype were $80.18,48.65$, and $36.04 \%$, respectively, with no significant difference $(\mathrm{P}=0.365)$. hOGG1, human 8 -hydroxyguanine glycosidase 1; NSCLC, non-small cell lung cancer.

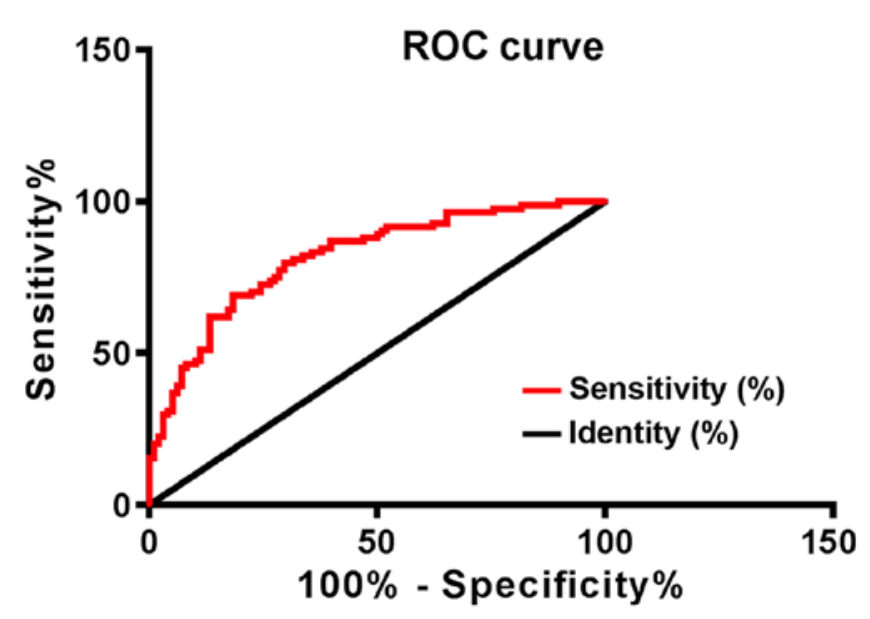

Figure 4. Predictive value of hOGG1 expression in the survival of patients with NSCLC. The sensitivity, specificity and AUC of hOGG1 in survival prediction were $83.33 \%, 64.29 \%$, and 0.816 , respectively. hOGG1, human 8-hydroxyguanine glycosidase 1; NSCLC, non-small cell lung cancer.

Expression and gene polymorphism of hOGGl and prognosis of NSCLC patients. Patients were followed up for 3 years by telephone, review or letter, to the last date of follow up. i.e., December 2017 or if patients died. The median value of the hOGG1 expression level in detection results as the boundary, NSCLC patients were divided into hOGG1 high expression group ( $\geq 3.61)$ with 91 cases and hOGG1 low expression group (<3.61) with 91 cases. The 1-, 2- and 3-year survival rates of patients in hOGG1 low expression group were 87.91, 70.33, and $63.74 \%$, respectively. Those of patients in hOGG1 high expression group were $79.12,56.04$, and $43.96 \%$, respectively. Those of patients in hOGG1 low expression group were significantly higher than those in hOGG1 high expression group $(\mathrm{P}=0.007)$. The number of $\mathrm{S} / \mathrm{S}+\mathrm{S} / \mathrm{C}$ was small, so they were merged into one group. Those of patients with hOGG1 rs1052133 of S/ $\mathrm{S}+\mathrm{S} / \mathrm{C}$ genotypes were $83.10,52.11$, and $45.07 \%$, respectively, and those of patients with $\mathrm{C} / \mathrm{C}$ genotype were $80.18,48.65$, and $36.04 \%$, respectively, with no significant difference $(\mathrm{P}=0.365)$ (Figs. 2 and 3).

Predictive value of hOGG1 expression in survival of patients with NSCLC. At 3 years, 98 patients with NSCLC survived, and 
84 patients had died. Patients were seperated into the survival group and the death group according to the survival. The hOGG1 expression in the survival group was $3.32 \pm 0.34$, and the hOGG1 expression in the death group was $3.81 \pm 0.39$. The sensitivity, specificity and AUC of hOGG1 for survival prediction were $83.33 \%, 64.29 \%$, and 0.816 , respectively (Fig. 4).

\section{Discussion}

Substantial data has shown that hOGG1 polymorphism is closely related to a variety of malignant tumors, such as bladder cancer, breast cancer and rectal cancer $(14,15)$. Therefore, the hOGG1 gene polymorphism and the prognosis were analyzed. First, the Hardy-Weinberg equilibrium analysis was performed on the hOGG1 genotype distribution frequency in NSCLC group and control group of patients. The results showed that there was no difference in the gene frequency between them. The genotypes of hOGG1 polymorphism locus rs1052133 in NSCLC group and control group included S/S, $\mathrm{S} / \mathrm{C}$, and $\mathrm{C} / \mathrm{C}$ genotypes. In NSCLC group of patients, there were 6 cases of S/S type, 65 cases of $S / C$ type and 111 cases of $\mathrm{C} / \mathrm{C}$ type. In control group of patients, there were 15 cases of S/S type, 90 cases of S/C type and 95 cases of $\mathrm{C} / \mathrm{C}$ type. Compared to $\mathrm{S} / \mathrm{S}$ and $\mathrm{S} / \mathrm{C}$ genotypes, the $\mathrm{C} / \mathrm{C}$ gene was found to be more common in NSCLC group than in control group. However, there was no significant difference in the 1-, 2- and 3 -year survival rates of patients with hOGG1 rs1052133 of $\mathrm{S} / \mathrm{S}+\mathrm{S} / \mathrm{C}$ genotypes and $\mathrm{C} / \mathrm{C}$ genotypes. The mutation of hOGG1 gene forms Ser326 and Cys326 results in low ability of hOGG1 to repair 8-hydroxy-deoxyguanine (8-oxoG). Having high mutagenicity, 8-oxoG is closely related to the occurrence and development of tumors, leading to an increased risk of cancers (16). Studies have reported (17) that when hOGG1 repairs DNA damage caused by 8 -oxoG, it is found that the repair ability of the $\mathrm{C}$ allele is approximately 7 times lower than that of the S allele. According to reports by Wang et al (18), people in Southeast Asia who carry C/C genotype have a significantly higher risk of developing liver cancer than those who carry S/C or S/S genotypes. According to Xie et al (19), the risk of death in patients with nasopharyngeal carcinoma with hOGG1 mutant genotype will be significantly higher than that of wild genotypes. Their results are consistent with the results of this study, which further support our experiments. It is indicated that rs1052133 locus can be used for NSCLC gene screening, but is not related to survival prognosis.

A retrospective analysis of clinical data of 182 NSCLC patients and 200 healthy individuals was performed. The results of RT-qPCR detection showed that the expression level of hOGG1 was significantly higher in cancer tissues than that in adjacent tissues in NSCLC group of patients, with a statistically significant difference. The median value of the hOGG1 expression level in detection results as the boundary, NSCLC patients were divided into hOGG1 high expression group and hOGG1 low expression group. The 1-, 2- and 3 -year survival rates of patients in hOGG1 low expression group were significantly higher than those in hOGG1 high expression group. AUC of hOGG1 expression in the survival prediction of patients with NSCLC was 0.816 which is a high predictive value. Studies have reported that the expression of most oncogenes and tumor suppressor genes can affect the proliferation and differentiation of tumor cells, correlated with the occurrence and development and prognosis of tumors (20). As a glycosidase that repairs oxidative DNA damage in humans, hOGG1 is closely related to the maintenance of the normal function of human genes and the occurrence and development of cancers (21). hOGG1 gene polymorphism has been reported to be important in a variety of digestive system cancers, closely related to the prognosis of various cancers. hOGG1 gene can inhibit the proliferation of lung cancer cells and the growth of lung cancer tumors (22). According to studies by Zhou et al (23), the hOGG1 expression in serum of patients with acute leukemia depression is higher than that in normal patients, which further supports our experimental results.

There are still some shortcomings in this study. For example, the specimen number selected for the study is small, due to limited experimental conditions. In addition, there may be differences in the hOGG1 gene polymorphism in NSCLC patients in different regions. Moreover, individual differences after treatment and intervention were not taken into consideration.

In summary, hOGG1 is highly expressed in NSCLC tissues. Compared to $\mathrm{S} / \mathrm{S}$ and $\mathrm{S} / \mathrm{C}$ genotypes, the $\mathrm{C} / \mathrm{C}$ gene was found to be more common in NSCLC group than in control group. hOGG1 has a high predictive value for patient survival. More studies on this polymorphism locus will be carried out in subsequent studies to determine whether this polymorphism can serve as a molecular marker for the risk of NSCLC.

\section{Acknowledgements}

Not applicable.

\section{Funding}

No funding was received.

\section{Availability of data and materials}

The datasets used and/or analyzed during the current study are available from the corresponding author on reasonable request.

\section{Authors' contributions}

JW drafted the manuscript. JW and PW conceived and designed the study. PW performed RT-qPCR and genotyping. JW and PW were equal contributors in extraction of total DNA and RNA. Both authors read and approved the final manuscript.

\section{Ethics approval and consent to participate}

This study was approved by Xiang Yang No. 1 People's Hospital, Hubei University of Medicine (Xiangyang, China). Signed informed consents were obtained from the patients and/or guardians.

\section{Patient consent for publication}

Not applicable. 


\section{Competing interests}

The authors declare that they have no competing interests.

\section{References}

1. Fehrenbacher L, Spira A, Ballinger M, Kowanetz M Vansteenkiste J, Mazieres J, Park K, Smith D, Artal-Cortes A, Lewanski C, et al; POPLAR Study Group: Atezolizumab versus docetaxel for patients with previously treated non-small-cell lung cancer (POPLAR): A multicentre, open-label, phase 2 randomised controlled trial. Lancet 387: 1837-1846, 2016.

2. Masters GA, Johnson DH and Temin S: Systemic therapy for stage IV non-small-cell lung cancer: American Society of Clinical Oncology Clinical Practice Guideline Update. J Oncol Pract 12: 90-93, 2016.

3. Jamal-Hanjani M, Wilson GA, McGranahan N, Birkbak NJ, Watkins TBK, Veeriah S, Shafi S, Johnson DH, Mitter R, Rosenthal R, et al; TRACERx Consortium: Tracking the evolution of non-small-cell lung cancer. N Engl J Med 376: 2109-2121, 2017

4. Gettinger S, Rizvi NA, Chow LQ, Borghaei H, Brahmer J, Ready N, Gerber DE, Shepherd FA, Antonia S, Goldman JW, et al Nivolumab monotherapy for first-line treatment of advanced non-small-cell lung cancer. J Clin Oncol 34: 2980-2987, 2016.

5. Planchard D, Yokoi T, McCleod MJ, Fischer JR, Kim YC, Ballas M, Shi K and Soria JC: A phase III study of durvalumab (MEDI4736) with or without tremelimumab for previously treated patients with advanced NSCLC: Rationale and protocol design of the ARCTIC study. Clin Lung Cancer 17: 232-236.e1, 2016 .

6. Emdad L, Lebedeva IV, Su ZZ, Gupta P, Sarkar D, Settleman J and Fisher PB: Combinatorial treatment of non-small-cel lung cancers with gefitinib and Ad.mda-7 enhances apoptosisinduction and reverses resistance to a single therapy. J Cell Physiol 210: 549-559, 2007.

7. Cravens SL and Stivers JT: Comparative effects of ions, molecular crowding, and bulk DNA on the damage search mechanisms of hOGG1 and hUNG. Biochemistry 55: 5230-5242, 2016.

8. Kadıŏlu E, Taçoy G, Özçağlı E, Okyay K, Akboğa MK, Cengel A and Sardaş S: The role of oxidative DNA damage and GSTM1, GSTT1, and hOGG1 gene polymorphisms in coronary artery disease risk. Anatol J Cardiol 16: 931-938, 2016.

9. Alanazi M, Pathan AAK, Shaik JP, Alhadheq A, Khan Z, Khan W, Al Naeem A and Parine NR: The hOGG1 Ser326Cys gene polymorphism and breast cancer risk in Saudi population. Pathol Oncol Res 23: 525-535, 2017.

10. Sliwinska A, Kwiatkowski D, Czarny P, Toma M, Wigner P, Drzewoski J, Fabianowska-Majewska K, Szemraj J, Maes M, Galecki P, et al: The levels of 7,8-dihydrodeoxyguanosine (8-oxoG) and 8-oxoguanine DNA glycosylase 1 (OGG1) - A potential diagnostic biomarkers of Alzheimer's disease. J Neurol Sci 368: 155-159, 2016

11. Li Z, Guan W, Li MX, Zhong ZY, Qian CY, Yang XQ, Liao L, Li ZP and Wang D: Genetic polymorphism of DNA base-excision repair genes (APE1, OGG1 and XRCC1) and their correlation with risk of lung cancer in a Chinese population. Arch Med Res 42: 226-234, 2011.
12. Cao WJ, Lu JZ, Li C, Gao YJ, Lu KQ, Wang HZ and Wang ZP: The hOGG1 Ser326Cys gene polymorphism and susceptibility for bladder cancer: A meta-analysis. Int Braz J Urol 42: 883-896, 2016.

13. Livak KJ and Schmittgen TD: Analysis of relative gene expression data using real-time quantitative PCR and the 2(-Delta Delta C(T)) method. Methods 25: 402-408, 2001.

14. Sanjari Moghaddam A, Nazarzadeh M, Bidel Z, Karamatinia A, Darvish $\mathrm{H}$ and Mosavi Jarrahi A: hOGG1 gene polymorphism and breast cancer risk: A systematic review and meta-analysis study. Breast J 24: 70-73, 2018.

15. Zhang M and Mo R: Association of hOGG1 Ser326Cys polymorphism with colorectal cancer risk: An updated meta-analysis including 5235 cases and 8438 controls. Tumour Biol 35: 12627-12633, 2014.

16. Lukina MV, Kuznetsova AA, Kuznetsov NA and Fedorova OS: The kinetic analysis of recognition of the damaged nucleotides by mutant forms of the 8-oxoguanine DNA glycosylase hOGG1. Russ J Bioorganic Chem 43: 1-12, 2017.

17. Kohno T, Shinmura K, Tosaka M, Tani M, Kim SR, Sugimura H, Nohmi T, Kasai H and Yokota J: Genetic polymorphisms and alternative splicing of the hOGG1 gene, that is involved in the repair of 8-hydroxyguanine in damaged DNA. Oncogene 16: 3219-3225, 1998

18. Wang W, Dang S, Li Y, Sun M, Jia X, Wang R and Liu J: hOGG1 Ser326Cys polymorphism and risk of hepatocellular carcinoma among East Asians: A meta-analysis. PLoS One 8: e60178, 2013.

19. Xie Y, Wu Y, Zhou X, Yao M, Ning S and Wei Z: Association of polymorphisms hOGGI rs1052133 and hMUTYH rs3219472 with risk of nasopharyngeal carcinoma in a Chinese population. Onco Targets Ther 9: 755-760, 2016.

20. Tan H: On the protective effects of gene SNPs against human cancer. EBioMedicine 33: 4-5, 2018.

21. Corella D, Ramírez-Sabio JB, Coltell O, Ortega-Azorín C, Estruch R, Martínez-González MA, Salas-Salvadó J, Sorlí JV, Castañer O, Arós F, et al: Effects of the Ser326Cys polymorphism in the DNA repair OGG1 gene on cancer, cardiovascular and all-cause mortality in the PREDIMED study: Modulation by diet. J Acad Nutr Diet 118: 589-605, 2018.

22. Wang Y, Gao X, Wei F, Zhang X, Yu J, Zhao H, Sun Q, Yan F, Yan C, Li H, et al: The hOGG1 Ser326Cys polymorphism contributes to digestive system cancer susceptibility: Evidence from 48 case-control studies. Tumour Biol 36: 1029-1038, 2015.

23. Zhou F, Zhang W, Wei Y, Zhou D, Su Z, Meng X, Hui L and Tian W: The changes of oxidative stress and human 8-hydroxyguanine glycosylase1 gene expression in depressive patients with acute leukemia. Leuk Res 31: 387-393, 2007.

This work is licensed under a Creative Commons Attribution-NonCommercial-NoDerivatives 4.0 International (CC BY-NC-ND 4.0) License. 